\title{
Fatigue lifetime prediction of a 3D-printing dental implant by Finite Element Analysis
}

\author{
Zeyuan Wang, Yufeng Zhou \\ School of Mechanical and Automotive Engineering, Shanghai University of Engineering Science 201620, China
}

\begin{abstract}
This study clarifies the differences between additive manufacturing and traditional manufacturing and the differences in product properties. The fatigue life of 3D printed dental implants was studied by finite element analysis. Pure titanium dental implant made by Concept laser3D printer in Germany is modelled by Solidworks 2016. Finite element analysis was performed in Ansys17.0 and the fatigue lifetime test is predicted in its post-processing system. These components are assembled by software Solidworks according to ISO14801. The maximum and minimum values of each index are obtained in the fatigue post process.
\end{abstract}

\section{Introduction}

Three-dimensional printed dental implants have been developed to meet the needs of different people. The impact and pressure on the implant during chewing can lead to fatigue failure. These fatigue failures can lead to a number of clinical complications. The life of dental implants has been reduced. For this reason, the fatigue properties of dental implants are required. Dental implants made from materials are still unstable in terms of internal mechanical properties due to metal powder accumulation. Therefore this study studies the fatigue performance of $3 \mathrm{~d}$ printed dental implants.

\section{Materials and methods}

\subsection{Three-dimensional Modelling}

The model used in this study is pure titanium dental implant made by Concept laser3D printer in Germany(Fig.1). In order to simulate the fatigue test process of dental implants, ISO14801 standard is required. According to the standard, endosseous dental implant from a system that includes only straight implant abutments shall be clamped such that its central longitudinal axis makes a $30^{\circ} \pm 2^{\circ}$ angle with the loading direction of the testing machine(Fig. 2). According to this standard, a two-stage dental implant is established. The structure of the common three-section dental implant has three parts: implant, abutment and screw. However, the implant used in this experiment only has two parts, namely the abutment screw and implant. [2] The abutment screw is the integrated structure of the abutment and screw. This structure increases the strength of the structure and makes assembly more convenient and easy. Using Solidworks2016, a 3d model of dental implant and test bench was established (Fig. 3).

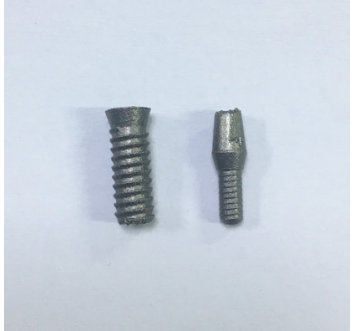

Figure 1. Three-dimensional dental implant

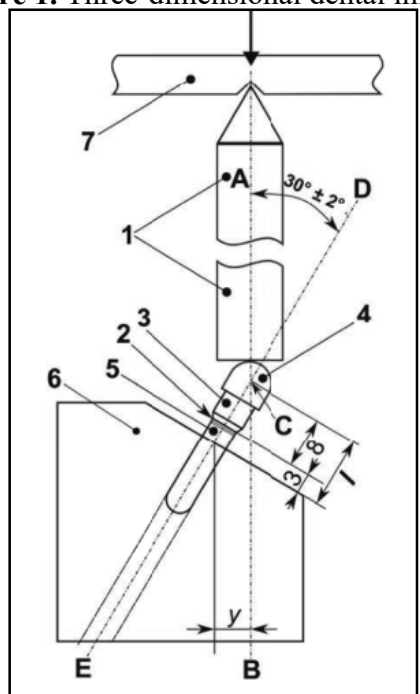

Figure 2. Below the figure. Schematic of test set-up for systems with no angulated implant abutments from ISO14801 


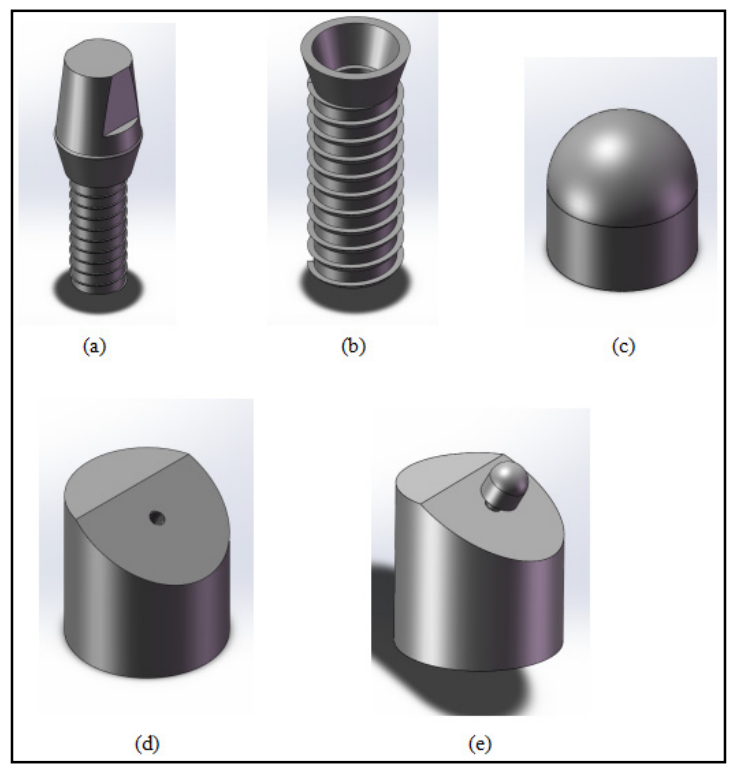

Figure 3. (a)Abutment screw; (b)implant body; (c)cap; (d)holder; (e)assembly.

The implant body length is $12 \mathrm{~mm}$.Its threaded part is $10 \mathrm{~mm}$, and the pitch is $1 \mathrm{~mm}$ (Fig. 3a). The abutment screw length is $12 \mathrm{~mm}$. The threaded part is $6 \mathrm{~mm}$ long and the pitch is $0.6 \mathrm{~mm}$ (Fig. 3b). The nylon cap (Fig. 3c) should be set on the base table in the testing process to prevent the fatigue testing machine from directly touching the surface of the implant and other failure cracks except fatigue cracks appear according to ISO14801. Figure 3(d) is the holding block of the simulated test bench in order to fix the tested implant. The holding block has an inclined plane at an Angle of 30 degrees from the horizontal axis. According to the standard ISO14801, an off-axis load with an Angle of 30 degrees is required to be loaded. The external thread of the abutment screw is matched with the external thread of the implant. This assembly is then matched with the thread of the holding block by the external thread of the implant. Finally, the protective nylon cap is put on the upper part of the abutment screw. In this way, the assembly was completed and the structure required for this test was obtained, as shown in Fig. 3(e). Save the assembly model built by solid works as a file in $\mathrm{x} \_\mathrm{t}$ format, and prepare to import it into $3 \mathrm{~d}$ finite element software for analysis.

\subsection{Properties of materials}

The performance of titanium parts produced by $3 \mathrm{~d}$ printing with pure titanium powder is very different from that produced by common machine. Therefore, the performance parameters of pure titanium cannot be used as experimental indexes. Different $3 \mathrm{~d}$ printing equipment produces parts with different performance. Even if the same equipment is in different environments (such as temperature, humidity, number of scans and processing efficiency, etc.), the parts produced will differ greatly in performance. But the difference had no material effect on the results. A series of material properties of this study were obtained through experiments. As shown in Table 1 1.104GPa young's modulus and 0.34 Poisson's ratio are assigned to the implant parts in the model. Young's modulus of $16 \mathrm{Gpa}$ and Poisson's ratio of 0.39 were assigned to the retention block material. In this study, all components of the material are considered uniform, linearly elastic and isotropic. The node on the base of the block is constrained to zero in three directions. This study will carry out fatigue postprocessing. Some data are only tested once, and the average value is not taken by repeated tests, so it is not very accurate, and there may be errors. It needs continuous improvement.[3]

Table 1. Material properties.

\begin{tabular}{|c|c|c|}
\hline & $\begin{array}{c}\text { Poisson's } \\
\text { ratio }\end{array}$ & $\begin{array}{c}\text { Young's } \\
\text { modulus (Gpa) }\end{array}$ \\
\hline Implant & 0.36 & 1.104 \\
\hline Holder & 0.39 & 16 \\
\hline
\end{tabular}

\subsection{Mesh Generation}

First built a component named mesh, and import the previously established $3 \mathrm{~d}$ model into the geometry. Then go to the mechanical edit interface. The model ontology without grid division can be seen in the interface. Create a grid, and select the details of the mesh in "Patch conforming method". Select the four sections that need to be divided into the grid : implant body, screw abutment, cap and holder. In this study, tetrahedrons method is used for the three-dimensional model. The algorithm includes patch conforming and patch independent and patch conforming is chosen. Global setting is used in element midside nodes[4]. Method includes automatic, tetrahedrons, hex dominant, sweep and multizone. According to model characteristics and analysis requirements, tetrahedrons are the most suitable method. The element type is C3D4 (tetrahedrons with four nodes), which is used for all components. The global mesh is refined continuously until the predicted maximum strain no longer varies with the increase of grid density[1]. The grid division of the model is completed as shown in figure 4

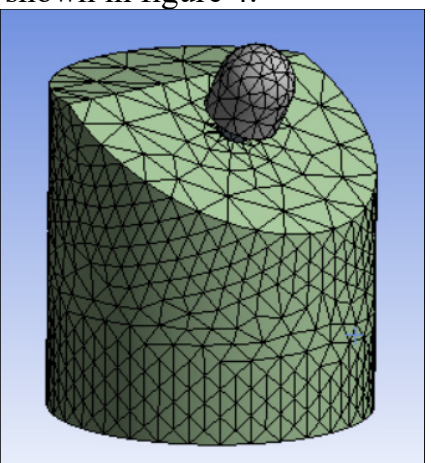

Figure 4. Mesh generation

\section{Results}

\subsection{Results of static analysis}

The node on the base of the block is constrained to zero in three directions[1]. Load a $30^{\circ}$ force that away from axial on the cap surface. The payload size is $200 \mathrm{~N}$. The 
three-dimensional model was analysed and the result of cloud map was obtained. Fig. 5(a) is the result of equivalent (von-mises) stress. The maximum value is $1.5215 \mathrm{E}+9 \mathrm{~Pa}$, which appears at the implant simulated bone level. The minimum value is $396.65 \mathrm{~Pa}$. Fig. 5(b) is the result of maximum principal stress. The maximum value was $9.8295 \mathrm{E}+8 \mathrm{~Pa}$, which appeared at the simulated bone level. Its minimum value is $2.2838 \mathrm{E}+8 \mathrm{~Pa}$. Fig. 5(c) is the results of maximum principal elastic strain. The maximum value is $4.7412 \mathrm{E}-5 \mathrm{~mm}$, which appears in the abutment screw.

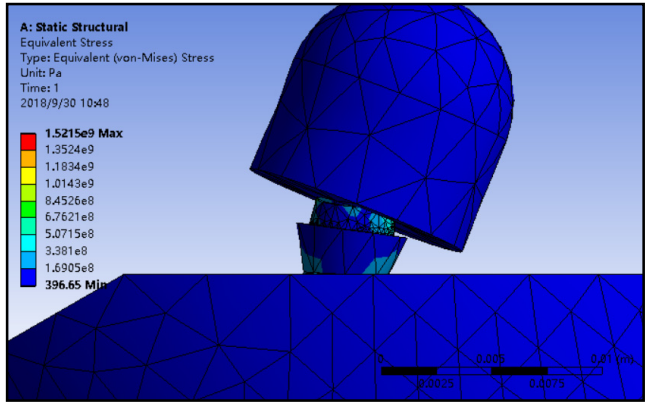

(a)

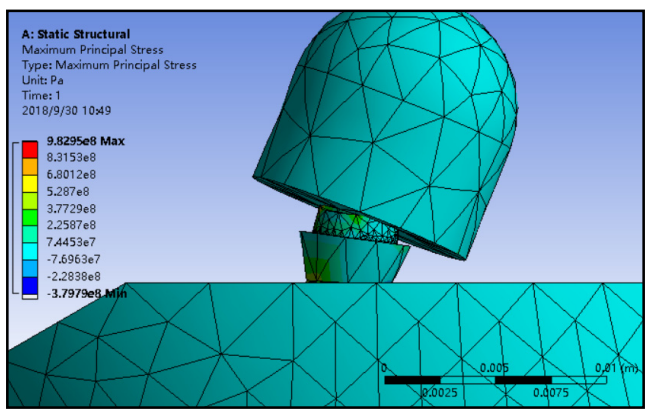

(b)

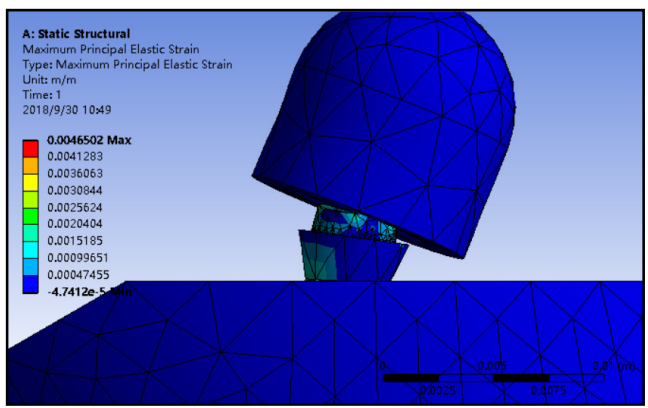

(c)

Figure 5. Mesh generation(a) equivalent (von-mises) stress; (b)maximum principal stress; (c)maximum principal elastic strain.

\subsection{Fatigue post-treatment}

Test loading was set as a cyclic load with a stress ratio (R) of 0.1 . This stress ratio was required by ISO 14801 to simulate the physiological chewing condition [1]. The fatigue post process of this study includes three definition type: life, safety factor and biaxiality indication. The model's four components are chosen to serve as life analysis process. It has no average across bodies. The minimum occurs on abutment screw, and its value is 84.001 cycles. The obtained cloud image results are shown in Fig 6(a). This curve result is shown on Fig. 7. All bodies are chosen in safety factor post processing. Initial design life is $1 \mathrm{E}+9$ cycles. The result is that the minimum occurs on abutment screw, and its value is 5.6656E-2. The obtained cloud image results are shown in Fig 6(b). This biaxiality indication analysis includes four all parts. Its maximum occurs on implant body and the value is 0.99448 . Its minimum occurs on abutment screw and the value is -0.99992 . The obtained cloud image results are shown in Fig 6(c).

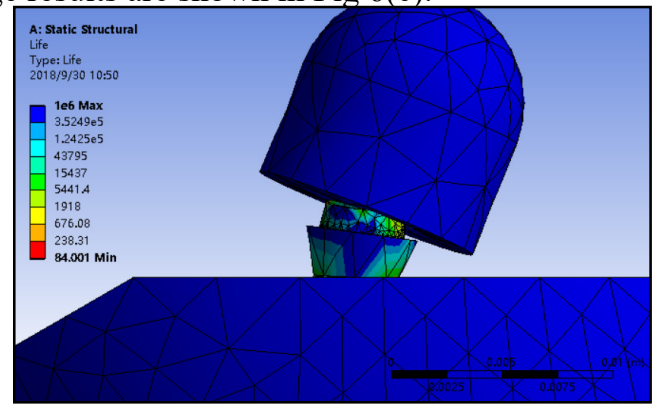

(a)

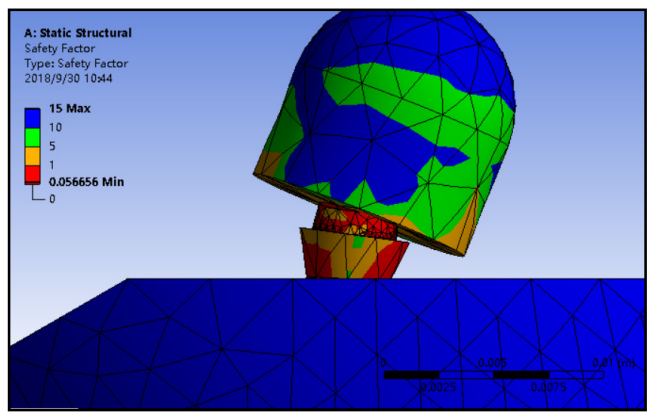

(b)

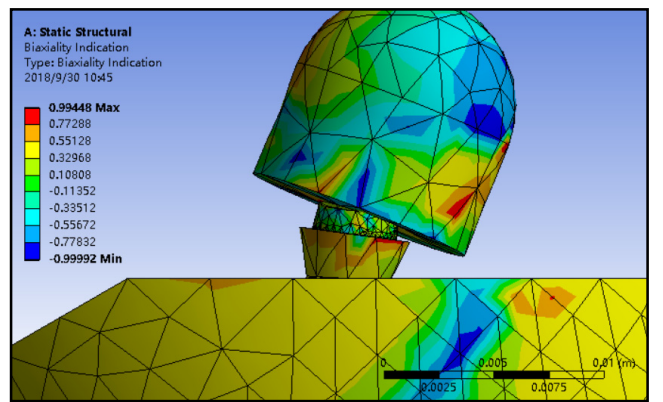

(c)

Figure 6. (a) Life; (b) safety factor; (c)biaxiality indication. 


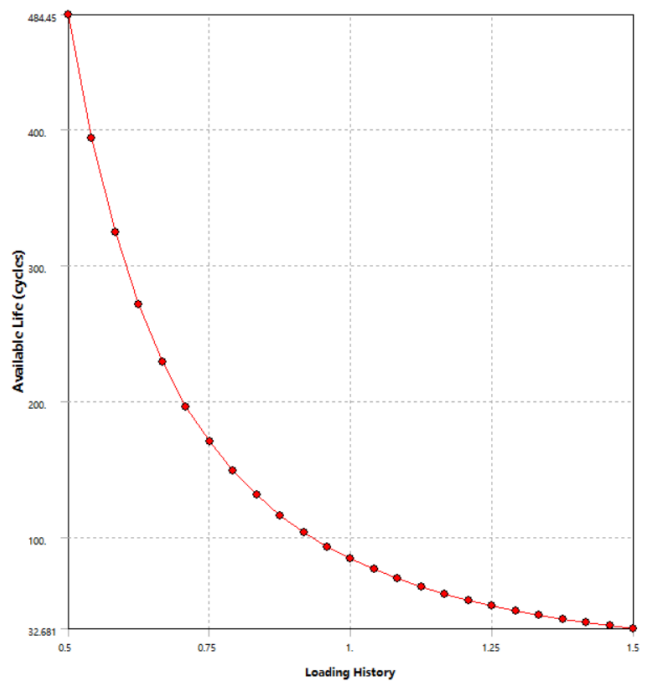

Figure 7. Available life curve

\section{Conclusions}

Fatigue treatment results of $3 \mathrm{~d}$ printed dental implants were obtained in this study. It can be used as a reference and further research is needed. Some of the data are only tested once, and there is no repeated test to take the average value, so it is not very accurate, and there may be errors. It needs continuous improvement. Different parts can be divided more carefully and rationally, which needs to be improved in the later stage. No fatigue test was conducted, and the fatigue characteristic data were calculated by formulas. Its accuracy needs to be improved.

\section{References}

1. Duan Y, Gonzalez J A, Kulkarni P A,DMOPADM 34, 9(2018)

2. Marchetti E, Ratta S, Mummolo S, Imp. Den. 23,6(2014)

3. Dhatrak P, Shirsat U, Deshmukh V,Pro.Fat, 73( 2018)

4. Jacobs R, Scarfe W C.DI, 43 (2018) 